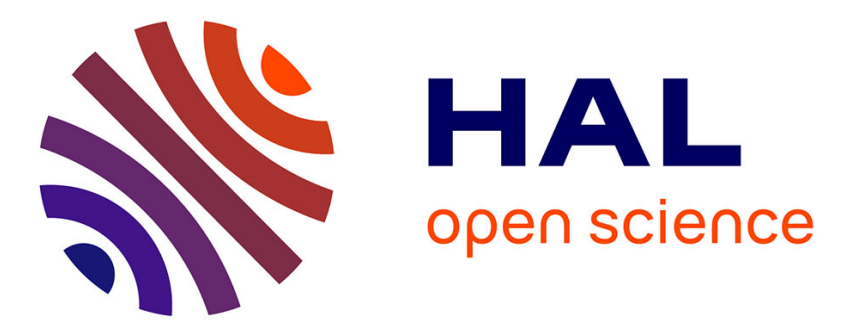

\title{
Etude de la précipitation du cuivre sous irradiation électronique dans des alliages dilués à base de fer
}

M. Mathon, F. Maury, A. Barbu, N. Smetniansky, N. Lorenzelli, C. de Novion, F. Boue

\section{- To cite this version:}

M. Mathon, F. Maury, A. Barbu, N. Smetniansky, N. Lorenzelli, et al.. Etude de la précipitation du cuivre sous irradiation électronique dans des alliages dilués à base de fer. Journal de Physique IV Proceedings, 1994, 04 (C3), pp.C3-193-C3-197. 10.1051/jp4:1994327 . jpa-00252524

\section{HAL Id: jpa-00252524 https://hal.science/jpa-00252524}

Submitted on 1 Jan 1994

HAL is a multi-disciplinary open access archive for the deposit and dissemination of scientific research documents, whether they are published or not. The documents may come from teaching and research institutions in France or abroad, or from public or private research centers.
L'archive ouverte pluridisciplinaire HAL, est destinée au dépôt et à la diffusion de documents scientifiques de niveau recherche, publiés ou non, émanant des établissements d'enseignement et de recherche français ou étrangers, des laboratoires publics ou privés. 


\title{
Etude de la précipitation du cuivre sous irradiation électronique dans des alliages dilués à base de fer
}

\author{
M.H. MATHON, F. MAURY, A. BARBU, N. SMETNIANSKY, N. LORENZELLI, \\ C.H. DE NOVION et F. BOUE*
}

Laboratoire des Solides Irradiés, Ecole Polytechnique, 91128 Palaiseau, France

* Laboratoire Léon Brillouin, CE/Saclay, 91191 Gif-sur-Yvette, France

\begin{abstract}
The copper precipitation in dilute ferritic alloys ( $\mathrm{FeCu}$ et $\mathrm{FeCuMn}$ ) has been studied under electron irradiation in the $170-370^{\circ} \mathrm{C}$ temperature range. The matrix depletion at low fluence has been followed by in situ electrical resistivity measurements, the mean radius and the number density of precipitates have been measured at higher fluence by Small Angle Neutron Scattering. The copper precipitation is not affected by manganese at $300^{\circ} \mathrm{C}$ but is strongly inhibited at $215^{\circ} \mathrm{C}$.

It is shown that a simple radiation enhanced precipitation model can reproduce quit well the first stage of precipitation but fails in the pure coarsening regime.
\end{abstract}

\section{INTRODUCTION}

La fragilisation des aciers de cuve des réacteurs à eau pressurisée, sous irradiation neutronique a $290^{\circ} \mathrm{C}$, se traduit par une augmentation de la température de transition ductile-fragile ainsi que par une perte de ductilité. Cette fragilisation et ce durcissement sont dus en partie à la précipitation des éléments résiduels présents dans la matrice sous forme d'ajouts $(\mathrm{Mn}, \mathrm{Ni}, \ldots)$ ou d'impuretés ( $\mathrm{Cu}, \mathrm{P}, \ldots)$. Le rôle prédominant du cuivre a été largement mis en évidence /1-3/, et notre étude s'attache à la compréhension des mécanismes de précipitation du cuivre seul ou en présence d'autres éléments. Nous avons étudié la précipitation du cuivre dans des alliages binaires et ternaires FeCu et EeCuMn irradiés avec des électrons de $2.5 \mathrm{MeV}$ d'énergie. L'irradiation électronique a pour avantage d'accélérer la diffusion du cuivre car la plupart des défauts créśs participent aux déplacements atomiques. Parallèlement, des recuits thermiques ont été effectués sur ces mêmes alliages afin d'établir une corrélation entre les mécanismes de précipitation obtenus sous irradiation ou vieillissement thermique.

La cinétique de précipitation du cuivre est suivie sous irradiation par mesure de résistivité électrique et les précipités sont caractérisés par Diffusion de Neutrons aux Petits Angles (DNPA).

\section{EXPERIENCES}

Echantillons:

Les études présentées ont êté réalisées sur deux alliages ferritiques $\mathrm{EeCu}$ et FeCuMn contenant (en poids) $1.5 \%$ de cuivre et $1.5 \%$ de manganèse. Ces alliages ont été élaborés par le LETRAM/SRMA (CESaclay). Les échantillons obtenus après laminage à froid jusqu'à une épaisseur de $400 \mu \mathrm{m}$ (irradiation) ou $800 \mu \mathrm{m}$ (vieillissement thermique), sont maintenus a $820^{\circ} \mathrm{C}$ pendant $24 \mathrm{~h}$ puis sont refroidis $\left(-10^{\circ} \mathrm{C} / \mathrm{s}\right.$ ) afin d'obtenir une solution homogène cubique centrée.

Irradiations:

Les alliages ont été irradiés avec des électrons de $2.5 \mathrm{MeV}$ jusqu'à des doses de $5 \mathrm{C} / \mathrm{cm}^{2}$ dans l'accelérateur Van de Graaff du Laboratoire des Solides Irradiés à des températures comprises entre $170^{\circ} \mathrm{C}$ et $370^{\circ} \mathrm{C}$. La cinétique de précipitation est suivie "in situ" par mesure à intervalles réguliers de la résistivité électrique à $30^{\circ} \mathrm{C}$. Dans l'hypothèse où les précipités n'influent pas sur la résistivité, la variation de la résistivité est directement lié à l'appauvrissement de la matrice en soluté /4/. 


\section{DNPA:}

Les expériences de DNPA ont été réalisées sur le spectromètre PAXY du Laboratoire Léon Brillouin (Laboratoire Commun CEA-CNRS) du CE-Saclay, à température ambiante, sous champ magnétique saturant $(\mathrm{H}=1.4 \mathrm{~T})$ et avec des neutrons de longueur d'onde de $6 \AA$.

L'intensité diffusée dépend des contrastes nucléaire et magnétique dus, respectivement, à la différence de composition et d'aimantation entre la matrice et les précipités. L'intensité des neutrons diffusés peut s'écrire en termes de section efficace $15 /$ :

$$
(\partial \Sigma / \partial \Omega)(q, \alpha)=f(1-f)\left[(\Delta \eta)^{2}{ }_{\text {nucl }}+(\Delta \eta)^{2}{ }_{\text {mag }} \sin ^{2} \alpha\right] F(q, h(R), S(q, R))
$$

où $q=4 \pi \sin \theta / \lambda$ est le vecteur de diffusion, $\alpha$ est l'angle entre l'aimantation de la matrice et le vecteur de diffusion, $f$ est la fraction volumique précipitée, $(\Delta \eta)_{i}^{2}$ sont les contrastes. $F(q, h(R), S(q, R))$ est le facteur de structure des particules diffusantes; il dépend du facteur de forme $S(q, R)$ des précipités considérés sphériques et de la distribution de taille des précipités $h(R)$ où $R$ est le rayon des précipités. La distribution de taille $h(R)$ est déterminée par ajustement des valeurs expérimentales.

L'utilisation d'un multidétecteur plan permet de mesurer simultanément l'intensité diffusée dans les directions parallèle $(\partial \Sigma / \partial \Omega)_{/ /}$et perpendiculaire $(\partial \Sigma / \partial \Omega)_{\perp}$ au champ magnétique appliqué et donc de séparer les composantes nucléaire et magnétique. Pour des systèmes simples, la composition des précipités est déduite du rapport $(A)$ des intensités mesurées dans les deux directions:

$$
\mathrm{A}=(\partial \Sigma / \partial \Omega)_{\perp} /(\partial \Sigma / \partial \Omega)_{/ /}
$$

\section{RESULTATS}

\section{1) Résistivité électrique:}

Les figures 1a et $1 \mathrm{~b}$ montrent les variations de la résistivité électrique avec la dose à différentes températures d'irradiation.
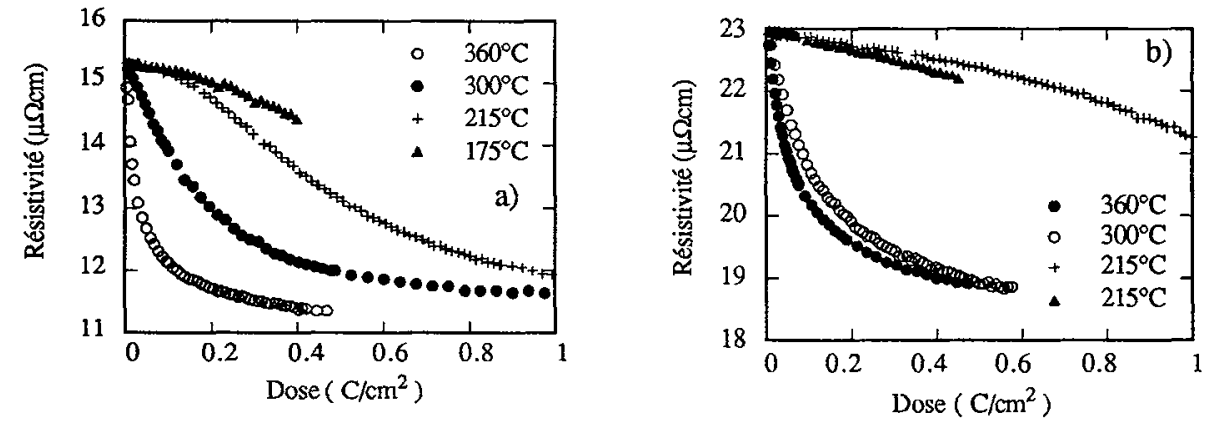

Fig1: Variation de la résistivité électrique avec la dose à différentes températures d'irradiation. (a):FeCu $1,5 \%$, (b) :FeCu $1,5 \% \mathrm{Mn}_{1,5 \%}$

La décroissance, rapide dès les faibles doses à haute température, résulte de l'appauvrissement en soluté de la matrice et peut être interprétée en terme de croissance des précipités. La saturation de la résistivité, observée à plus haute dose (au-delà de $0,5 \mathrm{C} / \mathrm{cm}^{2}$ à $\mathrm{T} \geq 300^{\circ} \mathrm{C}$ ) correspond à l'état où presque tout le cuivre est précipité et où l'évolution des précipités se fait uniquement par coalescence. $\mathrm{A} 300^{\circ} \mathrm{C}$, la présence de manganèse ne modifie pas sensiblement la cinétique de précipitation du cuivre. Par contre, l'évolution de cette cinétique avec la température d'irradiation est très différente dans les deux alliages:

- dans l'alliage $\mathrm{FeCu}$, la variation de résistivité enregistrée après $0,5 \mathrm{C} / \mathrm{cm}^{2}\left(\Delta \rho_{0.5}\right)$, varie linéairement avec la température d'irradiation;

- dans l'alliage FeCuMn, la cinétique de précipitation varie très peu entre 300 et $360^{\circ} \mathrm{C}$. Par contre, à basse température $\left(\mathrm{T} \leq 215^{\circ} \mathrm{C}\right)$, la précipitation du cuivre est extrêmement ralentie. En effet, $\left(\Delta \rho_{0.5}\right)$ représente seulement 10 à $15 \%$ de la variation totale obtenue à saturation contre $70 \%$ dans FeCu. Les 
variations de la résistivité enregistrées à $215^{\circ} \mathrm{C}$ pour deux échantillons différents, montrent que cet effet est parfaitement reproductible.

\section{2) DNPA:}

Les ajustements des résultats expérimentaux montrent que la distribution de taille des précipités est quasi-symétrique. En effet les meilleurs ajustements ont été obtenus avec une distribution gaussienne ou avec la distribution donnée par la théorie de Lishiftz-Slyosov-Wagner dans le cas d'une croissance gouvernée par les réactions d'interface /6-8/.

Les figures 2 et 3 représentent l'évolution du rayon moyen et du nombre par unité de volume des précipités en fonction de la dose d'irradiation à $300^{\circ} \mathrm{C}$ dans les deux alliages considérés.

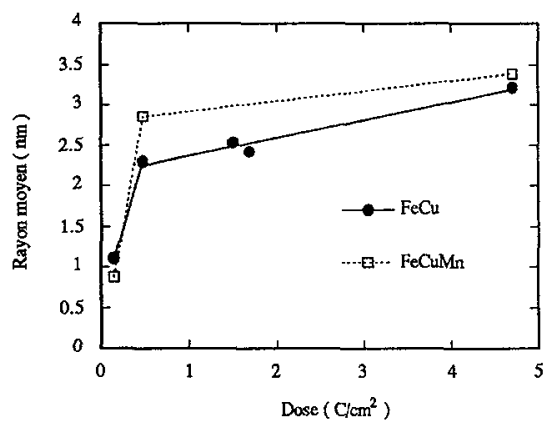

Fig 2: Evolution du rayon moyen des précipités en fonction de la dose à $300^{\circ} \mathrm{C}$

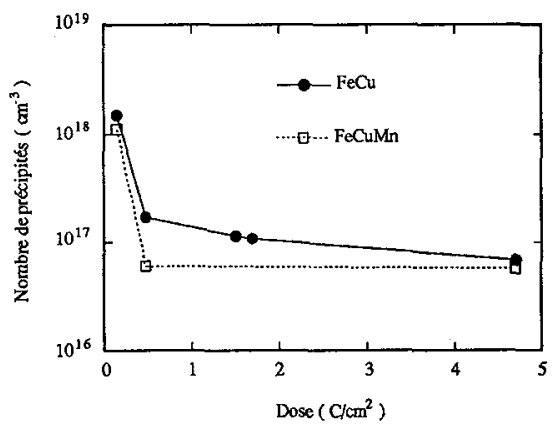

Fig 3: Evolution du nombre volumique des précipités en fonction de la dose à $300^{\circ} \mathrm{C}$

Cas de FeCu:

- A $300^{\circ} \mathrm{C}$, le rayon moyen des précipités augmente rapidement avec la dose alors que le nombre par unité de volume de précipités diminue â partir d'une faible valeur de la dose. Ceci montre que la coalescence commence très tôt, avant que la saturation en résistivité électrique soit atteinte.

- Les expériences réalisées à différentes températures montrent que pour une dose donnée $\left(0,5 \mathrm{C} / \mathrm{cm}^{2}\right)$ ( tableau 1$)$, le rayon moyen des précipités augmente linéairement avec la température d'irradiation.

- Le rapport A, caractérisant la composition des précipités, évolue avec la dose ( tableau 1). En effet pour les faibles doses, il est de l'ordre de 7,5 puis croît vers une valeur proche de 10 correspondant à des précipités composés de cuivre uniquement. Ce rapport est faible aussi dans le cas de courts vieillissements thermiques et d'irradiations de $0,5 \mathrm{C} / \mathrm{cm}^{2}$ à basses températures. Ce rapport dépend en fait de la taille des précipités et est nettement inférieur à 10 pour des précipités de petit rayon $(\mathrm{r}<1,5 \mathrm{ou} 2 \mathrm{~nm})$, tailles pour lesquelles les précipités sont cohérents avec la matrice (c.c.). L'abaissement de ce rapport peut être dû à la présence soit de lacunes ou dislocations, soit d'un gradient de concentration de fer à l'interface précipitématrice. Pour des forts vieillissements, le rapport rediminue. En effet, à partir d'un certain rayon moyen, les précipités deviennent incohérents (c.f.c.) avec la matrice $19 \%$. Les défauts ainsi formés à l'interface (dislocations) peuvent provoquer une diminution du rapport A par abaissement de la densité apparente.

\section{Cas de FeCuMn:}

- A $300^{\circ} \mathrm{C}$, l'évolution du rayon moyen et du nombre volumique de précipités avec la dose (Fig 2 et 3) est similaire dans les deux alliages; néanmoins le système parait évoluer plus rapidement aux faibles fluences dans FeCuMn. Par contre, à doses et rayons pratiquement équivalents, le rapport A est beaucoup plus faible dans l'alliage ternaire, ce qui est expliqué par la présence de 5 à $6 \%$ de manganèse dans la composition des précipités $/ 10 \%$. Ce rapport évolue aussi avec la taille des amas.

- A $215^{\circ} \mathrm{C}$, après une dose de $0,5 \mathrm{C} / \mathrm{cm}^{2}$, les précipités ne sont pas détectables en DNPA, donc trop petits. A basse température, la présence de manganèse semble inhiber la précipitation du cuivre. Ce phénomène peut résulter d'interactions entre le manganèse et les défauts ponctuels, non négligeables à basse température, qui diminueraient la mobilité des défauts ponctuels. 


\begin{tabular}{|c|c|c|c|c|}
\hline Alliage & $\begin{array}{c}\text { Température } \\
\left({ }^{\circ} \mathrm{C}\right)\end{array}$ & $\begin{array}{c}\text { Dose } \\
\left({\left.\mathrm{C} / \mathrm{cm}^{2}\right)}^{2}\right.\end{array}$ & $\begin{array}{c}\text { Rayon moyen } \\
(\mathrm{nm})\end{array}$ & $\begin{array}{c}\mathrm{A}= \\
(\partial \Sigma / \partial \Omega)_{\perp} /(\partial \Sigma / \partial \Omega),\end{array}$ \\
\hline $\mathrm{FeCu}$ & $300^{\circ} \mathrm{C}$ & 0,15 & 1,1 & 7,5 \\
\hline & & 0,50 & 2,3 & 10,2 \\
\hline & & 5,00 & 3,2 & 9,4 \\
\hline $\mathrm{FeCuMn}$ & $300^{\circ} \mathrm{C}$ & 0,15 & 0,9 & 3,2 \\
\hline & & 0,50 & 2,9 & 6,2 \\
\hline $\mathrm{FeCu}$ & & 5,00 & 3,4 & 4,8 \\
\hline & 175 & 0,5 & 1,2 & 6,2 \\
\hline & 360 & 0,5 & 1,45 & 6,5 \\
\hline
\end{tabular}

Tableau 1

\section{3) Modélisation de la précipitation du cuivre}

Si l'on veut être capable de prédire l'évolution de la fragilisation des aciers de cuve à temps long, il est important d'avoir un modèle de précipitation du cuivre sous irradiation.

On sait que l'effet de l'irradiation sur la précipitation peut a priori être de deux types:

1- Précipitation accélérée: l'irradiation a pour seul effet, par l'intermédiaire de la sursaturation de défauts ponctuels, d'accélérer la diffusion des espèces pondérales. La force motrice de précipitation est alors identique à celle qui est opérante lors d'un vieillissement thermique, à savoir le gain d'énergie libre entre l'état homogène et l'état précipité. Ce type de précipitation ne se produit évidemment que dans les solutions solides sursaturées et donne des microstructures identiques à celles qui sont observées lors de simples vieillissements thermiques.

2- Précipitation induite par les couplages entre les flux de défauts ponctuels et les flux de solutés /11/. Elle s'observe aussi bien dans les solutions solides sursaturées que dans les solutions solides sous saturées. La force motrice n'est plus de nature thermodynamique. Les microstructures observées sont généralement différentes de celles observées lors de vieillissements thermiques.

Nous avons montré que le premier mécanisme reproduit convenablement les courbes de résistivité électrique obtenues lors de la précipitation aussi bien sous irradiation entre $170^{\circ} \mathrm{C}$ et $370^{\circ} \mathrm{C}$ que lors de vieillissement thermique entre $390^{\circ} \mathrm{C}$ et $500^{\circ} \mathrm{C} / 13 /$.
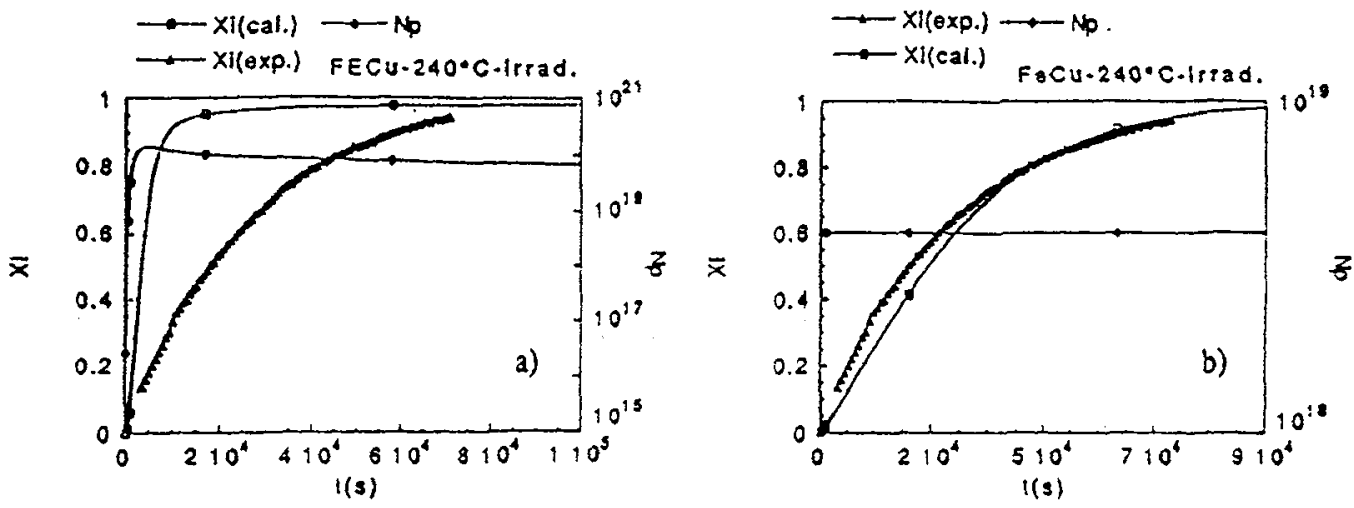

Fig4 : Évolution de l'avancement de la précipitation Xi déduite de la résistivité électrique et du nombre volumique de précipités $\mathrm{Np}$ sous irradiation à $240^{\circ} \mathrm{C}$

a) Dans l'hypothèse où tous les atomes de soluté sont sous forme de monomères à $t=0$

b) Dans l'hypothèse où $5 \%$ des atomes de soluté sont précipités à $t=0$

Le modèle utilisé, dû à Langer et Schwartz $/ 12 /$, prend en compte simultanément la germination, la croissance et la coalescence et ne considère que le premier moment de la distribution des précipités, c'est à dire leur taille moyenne. Deux conditions initiales différentes ont été considérées: dans la première, la solution est parfaitement homogène ( tous les atomes de soluté sont sous forme de monomères) alors que 
dans la seconde, $5 \%$ des atomes de soluté sont précipités à l'instant initial sous la forme de No précipités $/ \mathrm{cm}^{3}$ de rayon Ro.

Les paramètres sont le coefficient de diffusion du soluté $\mathrm{Db}$, la sursaturation en soluté et l'énergie d'interface dont la valeur est calculée dans l'approximation du champ moyen aux premiers voisins avec une énergie de ségrégation extraite du diagramme de phase en supposant que le système FeCu se comporte bien comme une solution régulière $/ 13 /$.

Les essais d'ajustement des courbes expérimentales de résistivité n'ont été possibles qu'avec la seconde hypothèse sur les conditions initiales (fig 4), mais il existe une infinité de solutions correspondant à des couples $(\mathrm{Db}, \mathrm{No})$ différents. Cependant, tous les couples n'ont pas une signification physique. Il est clair que hors irradiation, $\mathrm{Db}$ doit se situer dans la fourchette des valeurs expérimentales obtenues pour la diffusion du cuivre dans le fer. On en déduit que No doit se situer entre $10^{17}$ et $5.10^{18}$. Les valeurs de rayons moyens mesurées en DNPA permettent d'affiner encore la valeur de No. On trouve que sa valeur doit se situer autour de $4.10^{17} \mathrm{~cm}^{-3}$. On doit cependant remarquer que le modèle donne une vitesse de coalescence plus lente que celle qui est observée par DNPA (les mesures de résistivité sont pratiquement insensibles à la coalescence puisque celle-ci s'effectue à concentration de soluté dans la matrice pratiquement constante).

La raison pour laquelle il faut supposer que tous les précipités sont présents à l'instant initial provient de ce que la force motrice de précipitation est si élevée qu'il se produit un début de précipitation pendant la trempe.

\section{CONCLUSIONS}

Les mesures de résistivité électrique et les expériences de diffusion de neutrons aux petits angles ont permis de suivre la précipitation du cuivre dans les deux alliages Fe Cu et FeCuMn ainsi que de mettre en évidence l'effet du manganèse:

- à $300^{\circ} \mathrm{C}$ : le manganèse rentre un peu dans la composition des précipités mais n'influe pas sur la cinétique de précipitation du cuivre. Dans les deux alliages, la coalescence débute très tôt et le rapport $\mathrm{A}$, lié à la composition des précipités, évolue avec la taille de ces derniers;

- à basse température $\left(\mathrm{T} \leq 215^{\circ} \mathrm{C}\right)$, le manganèse inhibe la précipitation du cuivre.

D'autre part, nous avons montré qu'avec un modèle basé sur les hypothèses suivantes:

1- le seul effet de l'irradiation est d'accélérer la diffusion du soluté en augmentant la concentration de défauts ponctuels,

2- tous les germes sont présents à l'instant initial, c'est à dire après la trempe,

on peut reproduire les variations de résistivité obtenues sous irradiation entre 170 et $370^{\circ} \mathrm{C}$. Néanmoins, la coalescence est plus lente que celle observée expérimentalement par diffusion de neutrons aux petits angles.

\section{Remerciements:}

Nous tenons à remercier L. Noirez pour sa collaboration, P. Laplace et J.C. Bisson pour leur assistance technique.

\section{Références:}

/1/ Van Duysen J.C.,Rapport EDF, HT-41/PV D 724-A, 1990, EDF Les Renardières, 77250 Moret-surLoing, France

12/ Odette G.R., Scripta Met., 1983, 17, 1183

/3/ Russel K.C., Brown L.M., Acta Met., 1972, 20, 969

14/ Lê T.N., Barbu A., Liu D., F. Maury, Scripta Met., 1992, 26, 771-776

15/ Maury F., Mathon M.H., N. Lorenzelli, de Novion C.-H., Boué F., Gayraud N., J. de Phys. Colloque, même édition

/6/ Martin G., dans "Solid State Phase Transformation in Metals and Alloys", Aussois 1978, Les éditions de Physique, Orsay(1980), p.337

/7/ Lifshitz I.M., Slyozov V.V., J. Phys. Chem. Solids, 1961, 19, 35-50

18/ Wagner C., Z. Elektroch., 1961, 65, 581

19/ Pizzini S., Roberts K.J., Phythian W.J., English C.A., Greaves G.N., Phil Mag. Lett., 1990, 61, 223

/10/ Maury F., Lorenzelli N., de Novion C.H., J. Nucl. Mat.,1991, 183, 217-220

/11/ Barbu A., Martin G., Solid State Phenomena, 1993, 30 \& 31, 179-228

/12/ Langer J.S., Schwartz A.J., Phys. Rev., 1980, A21, 948

/13/ Smetniansky-de Grande N., Barbu A., à paraître 